\title{
Survey of obstetric and neonatal outcome in gestational diabetes mellitus
}

\author{
J. Princy Emil Josephine ${ }^{1 *}$, Susan William²
}

\author{
${ }^{1}$ Department of Obstetrics and Gynecology, Kanyakumari Medical College, Kanyakumari, Tamil Nadu, India \\ ${ }^{2}$ Clinical Director, William Hospital, Nagercoil, Tamil Nadu, India
}

Received: 08 September 2021

Revised: 13 October 2021

Accepted: 14 October 2021

\section{*Correspondence:}

Dr. J. Princy Emil Josephine,

E-mail: ogprincy80@gmail.com

Copyright: ( ) the author(s), publisher and licensee Medip Academy. This is an open-access article distributed under the terms of the Creative Commons Attribution Non-Commercial License, which permits unrestricted non-commercial use, distribution, and reproduction in any medium, provided the original work is properly cited.

\section{ABSTRACT}

Background: India being the diabetic capital of the world, Indian women have a high prevalence of gestational diabetes mellitus (16.5\%). It can cause a wide range of complications as well as long term implications in both the mother and fetus. A large proportion of women also progress to become overt diabetics in the future hampering with their quality of life by causing morbidity in various forms. Aim of this study is to evaluate the fetomaternal outcome in women with gestational diabetes mellitus at a tertiary care teaching hospital and the incidence of glucose intolerance in these women during the postpartum period.

Methods: All patients attending the antenatal OPD were offered a 75g glucose challenge test and 200 patients diagnosed with GDM were included in the study for a period of one year. Fetomaternal complications were studied and postpartum follow up was done in these patients.

Results: Out of these 200 women, $49 \%$ delivered via LSCS, $46 \%$ via labor naturalis and $5 \%$ via instrumental delivery. $59.5 \%$ were on insulin and $40.5 \%$ were treated with meal plan. Pre-eclampsia complicating pregnancy was seen in $26 \%$, polyhydramnios was encountered in $17.5 \%$, Urinary tract infection in $11 \%$, preterm labour in $8.5 \%$ and PROM in $7 \%$. Adverse fetal outcome was seen in 5\% of the babies. Birth asphyxia was seen in $7.5 \%$, macrosomia in $13 \%, 5 \%$ of the babies had congenital anomalies. In the postapartum follow up at 6 weeks $22.5 \%$ of the study population were glucose intolerant (75 gm OGTT).

Conclusions: Early detection and prompt management of this condition can tremendously reduce the short term and long-term complications in both the mother and fetus.

Keywords: Fetal outcome, Gestational diabetes mellitus, Maternal, Oral glucose tolerance test (OGTT)

\section{INTRODUCTION}

Gestational diabetes mellitus means hyperglycemia in pregnancy. By definition it is "carbohydrate intolerance with onset or first recognition during pregnancy". ${ }^{1}$ GDM is also defined as "carbohydrate intolerance with onset or recognition during pregnancy". ${ }^{1}$ GDM accounts for $\sim 90 \%$ of all pregnancies complicated by diabetes. ${ }^{1}$ GDM is associated with adverse outcome for the fetus and newborn (macrosomia, birth injuries, shoulder dystocia, respiratory distress syndrome, hypoglycemia, hyperbilirubinemia and childhood obesity). There is increased risk of gestational hypertension, preeclampsia, and operative delivery and their associated potential morbidities in women with GDM. ${ }^{1}$ More importantly, there is increased risk of developing type 2 diabetes mellitus (DM) in women diagnosed to have GDM with approximately $15 \%$ to $60 \%$ of them developing type $2 \mathrm{DM}$ within 5 to 15 years of delivery. ${ }^{2}$ Thus GDM offers a significant prospect for the development and application of clinical strategies for prevention of DM.

The prevalence of GDM varies significantly among different ethnicities, populations and with the diagnostic 
criteria used. Approximately $7 \%$ of all pregnancies in the United States are complicated by GDM, accounting for $>200,000$ cases per year. ${ }^{3}$ With the increase in obesity and sedentary lifestyle, the prevalence of GDM is increasing globally and more so in developing countries. ${ }^{1}$ In recent times there is increasing prevalence of pregnancies complicated by gestational diabetes mellitus. The worldwide prevalence ranges between $11-14 \%$. The prevalence is slightly higher in the Indian population $(16.5 \%)$, as we Indians are inherently more vulnerable to get affected owing toour hereditary and genetic makeup and ethnicity. ${ }^{2}$ In India, the prevalence of GDM is high and varies with geographical areas and diagnostic methods employed. The prevalence of GDM ranged from 3.8 to $21 \%$ in different parts of the India. ${ }^{4}$ GDM is more prevalent in urban areas than in rural areas. The prevalence of GDM was $2 \%$ in 1982 which increased to $7.62 \%$ in $1991 .^{5,6}$ The prevalence of GDM was $16.55 \%$ as per the random national survey conducted in 2002. The prevalence of GDM was $16.2 \%$ in the Chennai urban population. ${ }^{7}$ According to a community-based study, the prevalence of GDM varied in the rural, semi urban and urban areas. GDM was detected in $9.9 \%$ in rural, $13.8 \%$ in semi urban and $17.8 \%$ women in urban areas. ${ }^{8}$ Compared to Caucasian women, Indian women have an eleven-fold increased risk of having impaired glucose tolerance during pregnancy. ${ }^{9}$ Specific guidelines with recommendations for screening and diagnosing GDM have been issued by international and national medical organizations, along with expert committee and working groups. However, controversy concerning ideal strategy for the detection and diagnosis of GDM still continues. The issue of what is the best screening method for GDM remains unsettled. A universal recommendation for the optimal approach for screening and diagnosis of GDM remains obscure. Significant questions remain regarding the strategy for screening and diagnosis of GDM, the effect of diagnosis of GDM on the pregnant woman, her family and obstetric interventions in pregnancy, implications on health care costs and whether the diagnosis and treatment of GDM will improve meaningful maternal and neonatal outcome.

Despite the efforts which have been made in the understanding of DM and the availability of new therapeutic interventions, the pandemic of DM and its related complications continues unceasingly. There is an increase in GDM prevalence in all race/ethnicity as shown by studies conducted in different populations and with different methodologies. An increase in the prevalence of GDM aside from its adverse maternal and neonatal consequences, might reflect or contribute to the ongoing pattern of increasing DM and obesity. ${ }^{10}$ Universal screening for GDM identifies more cases and improves maternal and neonatal outcome. ${ }^{11}$ Hence universal screening for GDM is essential, as women of Asian origin and especially ethnic Indians, are at a greater risk of developing GDM and subsequent type 2 DM. For this, we need a simple procedure which is both feasible and economical. The one step World Health Organization (WHO) procedure using $75 \mathrm{gm}$ oral glucose tolerance test
(OGTT) to diagnose GDM serves both as a screening and a diagnostic modality at the same time. Hence, this study was undertaken to evaluate the prevalence of GDM using WHO criterion and its maternal and neonatal outcome.

\section{METHODS}

This was a done as a prospective study in department of obstetrics and gynaecology in Government Kanyakumari Medical College, Asaripallam for a period of one year from August 2019 to July 2020.

During the study period, 200 pregnant women irrespective of gestational age and risk factors were screened for GDM using $75 \mathrm{gm}$ oral glucose tolerance test (OGTT) and diagnosed to have GDM based on WHO criteria. Risk factors for GDM, maternal and neonatal outcomes were studied. Those who had GCT values more than $140 \mathrm{mg} / \mathrm{dl}$ were included in the study. Height, weight, and blood pressure were measured at every visit. Through proper history taking, clinical examination and lab investigations glycemic control was achieved on medical nutrition therapy or insulin and these patients were followed up from antenatal period till six weeks postpartum.

\section{Inclusion criteria}

Fetomaternal complications, perinatal outcome, the number of patients developing glucose intolerance postpartum (diagnosed by 75 gm OGTT) were evaluated during the study period.

\section{Exclusion criteria}

Patients with chronic hypertension, pregestational diabetes mellitus, patients on medications that can alter the glucose metabolism like steroids, antipyschotics, diuretics, oral contraceptive pills, beta blockers, patients with abnormal thyroid profile, Cushings syndrome, chronic medical illness, autoimmune disease, multiple pregnancy were excluded.

Study was done after getting approval from Institutional Human Ethics Committee.

\section{Statistical analysis}

Primary data was entered in MS Excel and analysed using SPSS 20v. The results were presented in terms of tables and graphs. The descriptive statistics frequency and percentage were calculated. The association between the categorical variables was analysed by chi square test with $5 \%$ level of significance.

\section{RESULTS}

In this study, the maximum population of GDM patients came under the age group 26-30 years $(32.5 \%)$. GDM in teenage pregnancy was encountered in $12.5 \%$ of the study population. The elderly gravidas covered $8 \%$. In this study, 
the occurrence of GDM was lesser in the extremes of age group. The lower incidence in the elderly could be probably because these mothers would have had established pregestational diabetes and therefore did not meet the inclusion criteria.

Majority of the study population delivered via lower segment caesarean section $(49 \%)$ out of which $21.2 \%$ had elective LSCS and $27.8 \%$ had emergency LSCS. The most common indications for emergency LSCS were the following with failed induction at $65.3 \%$ being most common, meconium-stained liquor- $15.2 \%$, foetal distress$15.5 \%$, cephalopelvic disproportion in labour- $14 \%$. Similarly, the indications for elective LSCS included macrosomia, repeat LSCS.

Table 1: Demographic features.

\begin{tabular}{|lll|}
\hline Characteristic & Frequency & Percent \\
\hline Age & & \\
\hline$<20$ years & 25 & 12.5 \\
\hline 21-25 years & 52 & 26 \\
\hline 26-30 years & 65 & 32.5 \\
\hline 31-35 years & 42 & 21 \\
\hline 36-40 years & 16 & 8 \\
\hline Mode of delivery & & \\
\hline LSCS & 98 & 49 \\
\hline Instrumental & 10 & 5 \\
\hline Labour naturalis & 92 & 46 \\
\hline Parity & & \\
\hline Primi & 87 & 43.5 \\
\hline G2P1L1 & 83 & 41.5 \\
\hline HOB & 30 & 15 \\
\hline
\end{tabular}

In our study $51 \%$ of the study population delivered vaginally out of which $46 \%$ delivered via labour naturalis and $5 \%$ via instrumental delivery (Table 1 ). The most common indication for instrumental delivery was large baby with birth weight more than $3.5 \mathrm{~kg}$. It was observed that in the study population, the caesarean section rate was higher in those women who were on insulin when compared to those on meal plan ( $\mathrm{p}$ value $=0.001$ ) which was statistically significant. Among the 92 women who delivered via labour naturalis, 47 of them were on insulin and 45 of them on meal plan. Equal proportion was also observed in the instrumental delivery.

Out of 200 women, 87 women were primi gravida $(43.5 \%) .83$ women were $2^{\text {nd }}$ gravidas with a previous live child $(41.5 \%)$. Higher order births constituted $15 \%$. In these two groups $80 \%$ had prior uncomplicated pregnancy and $20 \%$ had prior history of GDM, out of which $12 \%$ had normal OGTT value in the post-partum period and the remaining $8 \%$ did not turn up for the follow up. Women with previous history of abortion constituted $2 \%$ and in most of these women the cause of previous abortion was unknown.
The maximum occurrence of GDM was between 34-36 weeks of gestation $(54.2 \%)$, which can be attributed to the fact that insulin resistance was maximum during the last 3 months of pregnancy that was observed in the previous studies. $26.2 \%$ of GDM occurred between 28-34 weeks of gestation. The occurrence was $8.5 \%$ in gestation between 20-28 weeks and 7\% in gestation age more than 36 weeks. Only $4 \%$ of the study population were diagnosed with GDM at gestational age less than 20 weeks. In this study, 119 out of 200 women were started on insulin $(59.5 \%)$ and the remaining $81(40.5 \%)$ women had their glycemic control achieved with meal plan alone.

In this study, out of 200 women, 35 women had polyhydramnios. In these 35 women 22 were on insulin and 13 were on meal plan. Out of these 35,19 women had isolated polyhydramnios and the remaining 16 had other associated maternal complications.

Table 2: Incidence of preeclampsia.

\begin{tabular}{|llllll|}
\hline Preeclampsia & $\begin{array}{l}\text { Meal } \\
\text { plan }\end{array}$ & Insulin & Total & $\begin{array}{l}\text { Chi } \\
\text { sq. }\end{array}$ & $\begin{array}{l}\text { P } \\
\text { value }\end{array}$ \\
\cline { 1 - 4 } Absent & 69 & 79 & 148 & & \\
\cline { 1 - 4 } Mild & 5 & 13 & 18 & & \\
\cline { 1 - 4 } Severe & 7 & 27 & 34 & & 0.001 \\
\cline { 1 - 4 } Total & 81 & 119 & 200 & & \\
\hline
\end{tabular}

Pre-eclampsia was seen in $26 \%$ of the study population $(\mathrm{n}=52)$. Severe preeclampsia was seen in $17 \%(\mathrm{n}=34)$ and mild pre-eclampsia was seen in $9 \%(n=18)$. Among those who had severe preeclampsia $80 \%$ of them were on insulin and the remaining $20 \%$ were on meal plan. Among those who had mild preeclampsia $70 \%$ of them were on insulin and the remaining $30 \%$ were on meal plan (Table 2).

The incidence of pre-eclampsia was more in those women who were treated with insulin than those women whose glycemic control was achieved with meal plan alone and the $p$ value was statistically significant $(p$ value $=0.03 \mathrm{chi}$ $\mathrm{sq}=18.98$ ). This can be explained by the theory that insulin resistance also plays a role in pathogenesis of preeclampsia and the two conditions share common factors contributing to the pathogenesis.

Around $11 \%$ women had urinary tract infection. Out of these 16 women underwent preterm labour, giving birth to low weight babies. Most of UTI was seen in late half of $1^{\text {st }}$ trimester and the $2^{\text {nd }}$ trimester. In this study $8.5 \%$ had preterm labour. Most of them had associated complications of polyhydramnios and urinary tract infection indicating that these could be the causative factors for the preterm birth.

PROM was seen in $7 \%$ of the study population. Out of 200 women 14 had premature rupture of membranes. In these women most of them underwent caesarean section. Clear liquor was seen in 8 women who had PROM and the liquor was meconium stained in the remaining 6 . 
Out of the 200 GDM pregnancies, 190 were live birth $(95 \%)$. intra uterine death was seen in $2 \%(n=4) .1$ was still born $(0.5 \%)$ and early neonatal death (within 7 days) was seen in $2.5 \%$. The most common causes of early neonatal death were respiratory distress syndrome followed by hypoglycemia and HIE. Out of the 10 early neonatal death, 7 had congenital anomalies and the $p$ value was statically significant (Table 3 ).

Table 3: Fetal outcome.

\begin{tabular}{|lll|}
\hline Fetal outcome & Frequency & Percent \\
\hline IUD & 4 & 2 \\
\hline Still born & 1 & 0.5 \\
\hline Live birth & 190 & 95 \\
\hline Early neonatal death & 5 & 2.5 \\
\hline Total & 200 & 100 \\
\hline
\end{tabular}

All babies with the 10-minute APGAR less than 3 were said to have birth asphyxia and 15 babies $(7.5 \%)$ had birth asphyxia with the 10-minute APGAR score less than 3, of those 5 expired in the early neonatal period.

Most $(46.5 \%)$ of the babies born to GDM mothers had birth weight ranging from 2.5 to $3.5 \mathrm{~kg}(\mathrm{n}=93) .11 .5 \%$ of babies were low birth weight $(n=23)$. Of these 23 babies, 17 were preterm. $28.5 \%$ weighed between 3.6 to $4 \mathrm{~kg}$. Macrosomia (>4 kg) was seen in $13 \%$ of the babies. Birth trauma and shoulder dystocia was seen in 3 babies. Out of the 26 babies who were macrosomic, 24 were delivered by LSCS, 1 via instrumental and 1 via labour naturalis.

The incidence of congenital anomalies was seen in 5\% (10/200). $50 \%$ of these congenital anomalies occurred in early onset GDM), where the gestation age was less than 20 weeks. Intra uterine death was seen in 3 babies who had congenital anomalies. 3 anomalies babies suffered early neonatal death and 1 were still born. The remaining 3 babies had congenital anomaly compatible with life. The most common congenital anomaly encountered in order were single umbilical artery, spina bifida, septal defects, duodenal atresia.

Table 4: Post-partum follow up.

\begin{tabular}{|lll|}
\hline Post-partum & Frequency & Percent \\
\hline Normal & 144 & 72 \\
\hline Elevated & 45 & 22.5 \\
\hline Loss to follow up & 11 & 5.5 \\
\hline Total & 200 & 100 \\
\hline
\end{tabular}

In the post-partum follow up 200 GDM mothers the attrition rate was $5.5 \%(n=11)$. Of the remaining 189 women the OGTT was performed at 6 weeks post-partum and was found to be elevated in 45 women. In these 45 women, 35 women were on insulin and 11 women were on meal plan. The remaining 155 women had normal OGTT values. This implied that GDM women who were treated on insulin were glucose intolerant in the postpartum period as well and the $\mathrm{p}$ value was statistically significant. The ODDS ratio was 2.63 indicating that GDM women who were treated with insulin had twice the risk of becoming glucose intolerant than those on meal plan (Table 4).

\section{DISCUSSION}

GDM has been diagnosed as a clinical entity for the past 50 years. Early studies have strongly indicated untreated carbohydrate intolerance during pregnancy is associated with higher rates of maternal mortality and morbidity. The purpose of screening, treatment and management of GDM is to prevent still birth, congenital anomalies, preeclampsia, intra uterine death and decrease the incidence of macrosomic babies and cesarean section rates thereby reducing maternal and perinatal morbidity and mortality. The findings of the present study confirmed that GDM patients are liable to have adverse pregnancy outcomes.

The maximum incidence of GDM occurred between 26 to 30 years of age $(32.7 \%)$. Ismail et al reported the maximum mean maternal age of GDM in their study was 27.9 years. $^{12}$ The increasing incidence was seen in higher parity which was also reported by Farook et al. ${ }^{13}$ In this study similar findings were observed $(56.2 \%$ in multigravida and $43.8 \%$ in primi gravidas).

The maximum number of GDM cases were detected between 34 and 36 weeks of gestation $(54.2 \%)$, which can be attributed to the fact that the maximum insulin resistance occurs at this age which was also reinforced by Peraldi et al. ${ }^{14}$

Chanu et al studied that caesarean section rates were higher in women with GDM (52\%). In this study, the incidence of caesarean section was higher $(49 \%)$ when compared to labour naturalis $(46 \%){ }^{15}$

Ameya et al studied the feto maternal outcomes in GDM and found that preclampsia complicating pregnancy was found in $26 \%$ of GDM mothers. In this study also, $26 \%$ of GDM mothers had associated GDM complicating pregnancy. ${ }^{16}$

Chanu et al observed increasing frequency of preterm labour and polyhydramnios in GDM patients. ${ }^{15}$ Krishnamoorthy et al studied that the incidence of preeclampsia in GDM was 30\% and preterm labor and PROM was $9 \%$ and $8 \%$ respectively. ${ }^{17}$ In this study preterm labour was encountered in the population and PROM in $6.8 \%$.

As far as the fetal complications were concerened congenital anomalies were encountered in $5.2 \%$ of the study population, while according to Ameya et al $8 \%$ had congenital anomalies. ${ }^{16}$ The incidence of macrosomia was $13.2 \%$ in this study whereas higher incidence was noted in the other studies (40\% in study by Ameya et al and $23 \%$ in study by Mutummatou et al). ${ }^{15,16}$ Adverse fetal outcome (still born, intrauterine death, early neonatal death) was 
seen in $5 \%$ of the study population and birth asphyxia in $7.2 \%$.

During the postpartum follow up at six weeks, $22.8 \%$ of the women were glucose intolerant and those on insulin had twice the risk of being glucose intolerant than those on meal plan alone. Kjos et al performed 75 gm OGTT 5 to 8 weeks after delivery in 246 women with GDM and found $19 \%$ had abnormal OGTT, out of which $10 \%$ had impaired glucose tolerance and $9 \%$ had T2DM. ${ }^{18} 16.9 \%$ of the population were glucose intolerant in the study by Ameya et al. ${ }^{16}$

This study has some limitations. The GDM mothers could be followed up only till six weeks postpartum and long term follow up was not feasible in the one-year study period. Postpartum follow up could not be achieved in $100 \%$ of the study population as few women did not turn up for follow up.

\section{CONCLUSION}

To conclude, based on the observations of this study, GDM is associated with adverse complications in both the mother and fetus. A large proportion of women also progress to become overt diabetics in the future hampering with their quality of life by causing morbidity in various forms. Therefore, all antenatal women attending the OPD should be offered a simple Glucose challenge test and if found negative the test has to be repeated every trimester. Once diagnosed with GDM appropriate glycaemic control either via insulin or meal plan has to be achieved for good pregnancy outcome and to prevent the complications. Proper counselling should be given to the patient at the time of discharge to have her sugars checked in the postpartum period. Early detection and prompt management of this condition can tremendously reduce the short term and long-term complications in both the mother and neonate.

Funding: No funding sources Conflict of interest: None declared

Ethical approval: The study was approved by the Institutional Ethics Committee

\section{REFERENCES}

1. Gestational Diabetes Mellitus. ACOG Practice Bulletin \#137. American College of Obstetricians and Gynecologists. Obstet Gynecol. 2013;122:406-16.

2. Kim C, Newton KM, Knopp RH. Gestational diabetes and the incidence of type 2 diabetes: a systematic review. Diabetes Care. 2002;25:1862-8.

3. American Diabetes Association. Gestational diabetes mellitus. Diabetes Care. 2004;27(1):88-90.
4. Seshiah V, Balaji V, Balaji MS, Paneerselvam A, Kapur A. Pregnancy and diabetes scenario around the world: India. Int J Gynaecol Obstet. 2009;104(1):S35-8.

5. Agarwal S, Gupta AN. Gestational Diabetes. J Assoc Phys India.1982;30(4):203-5.

6. Narendra J, Munichoodappa C, Gurudas A, Ram Prasad AV, Madhav T, Vijayalakshmi, et al. Prevalence of glucose intolerance during pregnancy. Int J Diab Dev Countries. 1991;11:2-4.

7. Seshiah V, Balaji V, Balaji MS, Sanjeevi CB, Green A. Gestational diabetes mellitus in India. J Assoc Phys India. 2004;52:707-11.

8. Seshiah V, Balaji V, Balaji MS, Paneerselvam A, Arthi T, Thamizharasi M, Datta M. Prevalence of gestational diabetes mellitus in South India (Tamil Nadu) - a community based study. J Assoc Phys India. 2008;56:329-33.

9. Dornhorst A, Paterson CM, Nicholls JS, Wadsworth J, Chiu DC, Elkeles RS, et al. High prevalence of gestational diabetes in women from ethnic minority groups. Diabetes Med. 1992;9:820-5.

10. Ferrara A. Increasing prevalence of gestational diabetes mellitus- a public health perspective. Diabetes Care. 2007;30(2):S141-6.

11. Griffin ME, Coffey M, Johnson H, Scanlon P, Foley M, Stronge J, et al. Universal versus risk factor-based screening for gestational diabetes mellitus: detection rates, gestation at diagnosis and outcome. Diabetes Med. 2000;17(1):26-32.

12. Ismail NA, Aris NM, Mahdy ZA, Ahmad S, Naim NM, Siraj HH, Zakaria SZ. Gestational diabetes mellitus in primigravidae: a mild disease. Acta Medica (Hradec Kralove). 2011;54:21-4.

13. Farooq M, Ayaz A, Ali Bahoo L, Ahmad I. Maternal and neonatal outcomes in gestational diabetes mellitus. Int J Endocrinol Metab. 2007;5(3):109-15.

14. Catalano PM, Huston L, Amini SB, Kalhan SC. Longitudinal changes in glucose metabolism during pregnancy in obese women with normal glucose tolerance and gestational diabetes mellitus. Am J Obstet Gynecol. 1999;180:903-16.

15. Chanu M, Syiemleh AJ, Pradhan B, Devi RK. Clinical study of fetomaternal outcome of gestational diabetes mellitus. J Dent Med Sci. 2015;14(4):53-6.

16. Dudhwadkar AR, Fonseca MN. Maternal and fetal outcome in gestational diabetes mellitus. Int J Reprod Contracept Obstet Gynecol. 2016;5:3317-21.

17. Krishnamoorthy U, Schram CM, Hill SR. Maternal obesity in pregnancy: is it time for meaningful research to inform preventive and management strategies? BJOG. 2006;113(10):1134-40.

18. Kjos SL, Peters RK, Xiang A, Henry OA, Montoro M, Buchanan TA. Predicting future diabetes in Latino women with gestational diabetes: utility of early postpartum glucose tolerance testing. Diabetes. 1995;44(5):586-9.

Cite this article as: Josephine JPE, William S.

Survey of obstetric and neonatal outcome in gestational diabetes mellitus. Int J Reprod Contracept Obstet Gynecol 2021;10:4278-82. 